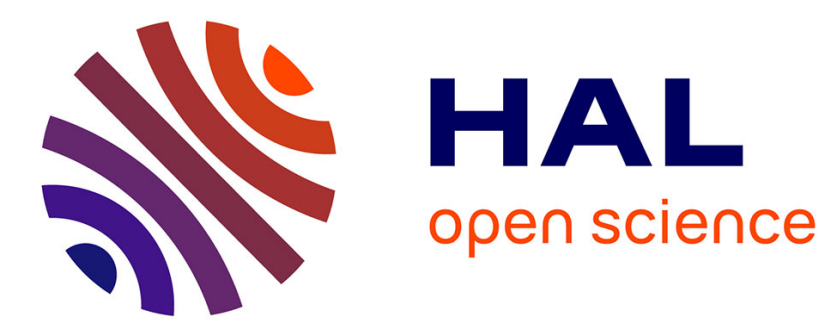

\title{
Modeling Airline Crew Activity to Improve Flight Safety Analysis
}

\author{
Nicolas Maille
}

\section{To cite this version:}

Nicolas Maille. Modeling Airline Crew Activity to Improve Flight Safety Analysis. 38th IEEE Aerospace Conference, Mar 2017, Big Sky, MT United States. hal-01383972

\section{HAL Id: hal-01383972}

\section{https://hal.science/hal-01383972}

Submitted on 19 Oct 2016

HAL is a multi-disciplinary open access archive for the deposit and dissemination of scientific research documents, whether they are published or not. The documents may come from teaching and research institutions in France or abroad, or from public or private research centers.
L'archive ouverte pluridisciplinaire HAL, est destinée au dépôt et à la diffusion de documents scientifiques de niveau recherche, publiés ou non, émanant des établissements d'enseignement et de recherche français ou étrangers, des laboratoires publics ou privés. 


\title{
Modeling Airline Crew Activity to Improve Flight Safety Analysis
}

\author{
Nicolas Maille \\ ONERA - The French Aerospace Lab \\ Base Aérienne 701. \\ 13661 Salon Air, France \\ (+33) 490-170-131 \\ Nicolas.Maille@onera.fr
}

\begin{abstract}
This paper reviews processes used by airlines to analyze inflight recorded parameters in their safety management system. Then it investigates how the expected crew activity can be modeled, based on airlines' standard operating procedures, and used to get a better understanding of operational risks. The language allowing the hierarchical description of the crew activity is described as well as the process dedicated to the identification of these elements from the recorded flight parameters. As an application case study, a go-around procedure is modeled and used to analyze a set of real aviation data. Results emphasize how this new flight data analysis process can complement existing tools.
\end{abstract}

\section{TABLE OF CONTENTS}

1. INTRODUCTION 1

2. FLIGHT DATA MONITORING .................................... 2

3. MODELING FLIGHT OPERATING PROCEDURES..... 3

4. APPLICATION TO GO-AROUND PROCEDURES ...... 6 5. NEW POSSIBILITIES FOR AVIATION SAFETY ANALYSIS 7

6. CONCLUSIONS 10

REFERENCES. 10

BIOGRAPHY 11

\section{INTRODUCTION}

Even if the global accident rate ${ }^{1}$ for 2015 , is the lowest recorded over the last few years (see Figure 1), safety remains a major challenge for regulators and air operators while the traffic is still increasing in a complex environment. A coordinated, structured, risk-based approach is essential to improve global aviation safety. At the airline level, one of the key elements is the development of Safety Management Systems (SMS) that identify a standardized, data driven system of hazards identifiers, risk assessment, and risk mitigation that enable highly complex systems to run [3].

The cornerstone of a successful SMS program is a proper risk assessment which is based on the estimate of severity and likelihood of life-threatening situations encountered in flights. The digital flight data available on all modern commercial aircraft represent a major source of information

${ }^{1}$ As calculated by the International Civil Aviation Organization (ICAO), cf. [1] and [2].

978-5090-1613-6/17//\$31.00 @2017 IEEE to acquire such an understanding of what happens during routine operations. For that matter, ICAO includes in their definition of SMS [4, Section 3.3.6] the need for flight data analysis and accumulation of these data. Also major airlines set up highly sophisticated data collection programs (called FOQA $^{2}$ or $\mathrm{FDM}^{3}$ program). Nevertheless, there is no instruction defining how the analysis has to be performed and existing tools cannot afford an efficient proactive safety management based on the identification of new threats. Thus, huge databases with highly valuable information about the operational conditions are available in airlines, but are under-exploited for predictive risk assessment. The development of new analysis methodologies that will complement existing FOQA tools is a step required for a real safety enhancement.

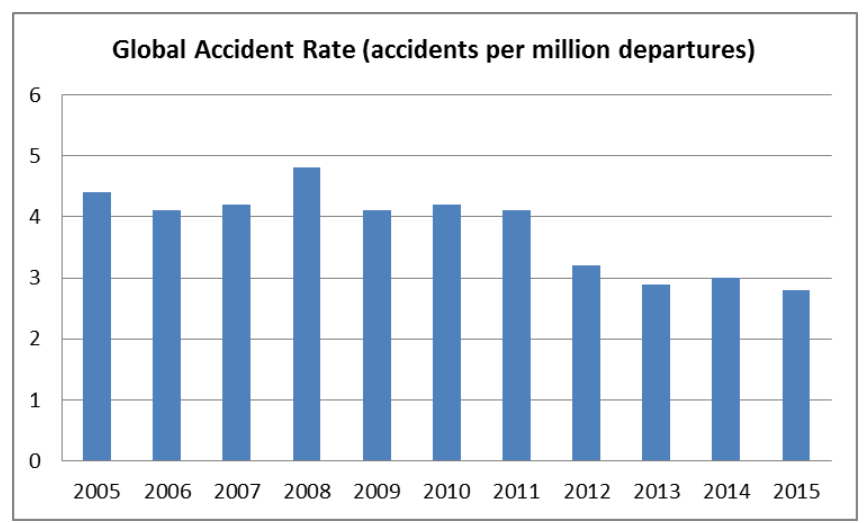

Figure 1: Global accident rate trends, based on scheduled commercial operations involving aircraft having a maximum take-off weight above $5700 \mathrm{~kg}$.

The main contribution of this paper is to investigate how to improve on the analysis process with a better use of airline's Standard Operating Procedures (SOPs). The standard methodology used in commercial FOQA tools and other approaches are described in section 2 of this paper. Section 3 presents how flight operating procedures can be modeled and identified from the temporal evolution of recorded flight parameters. In section 4 this framework is applied to real aviation data in order to describe and analyze go-around situations. Then, section 5 discusses how this new approach, based on the identification of crew activity can help to

\footnotetext{
${ }^{2}$ Flight Operations Quality Assurance.

${ }^{3}$ Flight Data Monitoring.
} 
improve aviation safety management. Section 6 provides conclusions and perspectives.

\section{Flight DAta Monitoring}

Major commercial airlines routinely download digital flight data recorded during flights, store and analyze them to manage safety. They handle a large number of flights ${ }^{4}$, and the process has to be highly automated. Worldwide, the most widely used FDM tools [5] (Teledyne AirFASE, Spirent GRAF, and Sagem AGS) primarily rely on the identification of prescribed exceedances that characterize events that fall outside operator-determined standards. Section 2.1 describes this exceedance based analysis process while the following one illustrates other underdevelopment approaches.

\subsection{Prescribed exceedances}

Recorded flight data are decoded and analyzed on the ground FDM workstation generating reports according to the safety limits imposed by the airline. The analysis process includes: (1) filtering and data cleaning, (2) triggering of safety events (when flight parameters values fall outside of the safety limits) and, (3) statistics on the identified events. The crucial point in this process is the definition of prescribed exceedances that characterizes safety events. Indeed, the accurate definition of these deviations from normal operations is a key element for a comprehensive flight data analysis [6]. Their characterization is generally realized in two stages. First, the flight is decomposed in flight phases, for example from taxiout to taxi-in. The number of possible flight phases and their starting conditions vary with the software, and starting conditions are usually based on combination of thresholds for some parameters.

As an example, in one tool, the Approach phase starts when:

1. The aircraft has been, at least for 10 seconds, below $4000 \mathrm{Ft}$ above ground level with a rate of descent over $420 \mathrm{Ft} / \mathrm{mn}$ and one engine below $90 \% \mathrm{~N} 2^{5}$.

2. When this first condition has been fulfilled, the Approach phase is validated if the aircraft is between $3000 \mathrm{Ft}$ and 1000Ft AGL (Above Ground Level) for at least 10s.

The set of rules defining all the possible flight phases is set by experts and is embedded in the tool. After this first stage, the flight is split up in successive flight phases (Figure 2).

The second stage is the triggering of the events. It relies on the definition of prescribed exceedances which capture deviations from normal operations that the safety department wants to identify and use as safety performance indicators. These unwanted situations are characterized by small sets of parameters that exceed some predefined

\footnotetext{
${ }^{4}$ Typically from 500000 up to 2000000 flights a year.

${ }^{5} \mathrm{~N} 1$ and $\mathrm{N} 2$ are the rotational speeds of the engine sections (N1 for low pressure compressor and $\mathrm{N} 2$ for the high pressure compressor) expressed as a percentage of the nominal value.
}

thresholds. Both the choice of parameters and thresholds rely on human expertise.

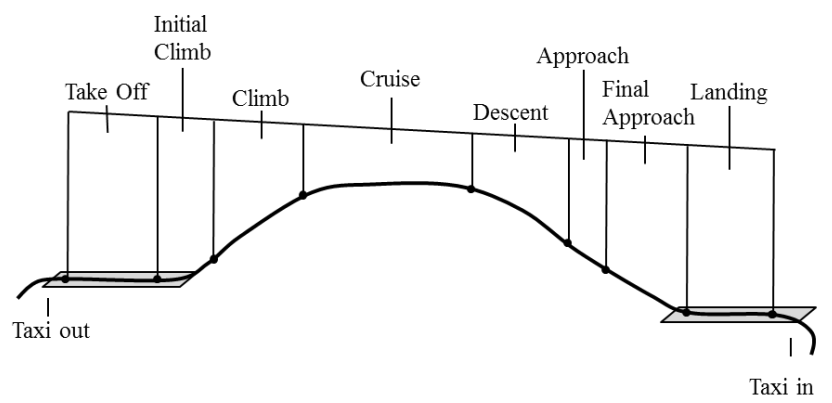

Figure 2: Example of flight phases.

For example the triggering of "Heading deviation at takeoff" events could be defined as follow:

(1) the flight phase is Take-OFF

(2) the Ground Speed of the aircraft is over $100 \mathrm{Kt}$ and the Pitch is under $1^{\circ}$

(3) the reference heading (HeadingRef) is the heading of the aircraft when it just goes over 100Kt during the Take-Off flight phase

(4) the current heading deviation (Dev) is calculated as the absolute value of the difference between the current heading of the aircraft and the heading Reference

$$
\operatorname{Dev}(\mathrm{t})=\operatorname{ABS}(\operatorname{Heading}(\mathrm{t})-\text { HeadingRef })
$$

(5) an event of high severity is triggered if the current heading deviation is over $4^{\circ}$ during $3 \mathrm{~s}$

else an event of medium severity is triggered if the current heading deviation is over $3^{\circ}$ during $3 \mathrm{~s}$

else an event of low severity is triggered if the current heading deviation is over $2.5^{\circ}$ during $2 \mathrm{~s}$

Rules (1) and (2) define the temporal window in which the exceedance will be searched for. Rules (3) and (4) set how the parameter, which has to be compared to prescribed thresholds, is calculated. Finally rule (5) describes the conditions required to trigger the events with an associated severity level.

The definition of all these rules is a complex task that requires lot of expertise. These sets of rules are usually built in the airline and completed when new issues are discovered [7]. A set of prescribed exceedances (often between 50 and 150 ) is defined for each fleet (as the thresholds will depend on the aircraft characteristics and performances). These exceedances concentrate on the final approach and landing phases which are known to be the most critical on a safety point of view (cf. [8] page 21: $49 \%$ of fatal accidents occurred during the final approach or the landing for worldwide commercial jet fleet from 2006 through 2015). This approach is well fitted to manage known safety issues that can be clearly specified thanks to operational deviations. Significant safety improvements have been done by airlines that routinely use these tools on all their flight 
data. The strength of this approach consists in the reliability of the search of these prescribed events on all the available flights. So an objective view of how flights comply with these specific deviations can be set up. Such tools are a major piece in a Safety Management System because it allows an objective valuation of the severity and the likelihood of safety events (such as un-stabilized approaches). Nevertheless these tools are limited to the search of known safety events and there is a flagrant need of complementing methodologies that help to discover new emerging risks, even if more complex definitions of events can be included in these tools [9].

\subsection{Data-mining}

The development of new research fields, such has "Knowledge Discovery in Database" or "data-mining", combined with the rapid increase of computing power offers new perspectives for the analysis of these flight data. Since the late 90's, guided by the will to find "the unknown" some data-mining methods have been specialized in the analysis of these flight data. The idea was to free of prescribed exceedances but to use large collections of data to identify abnormal situations. Clustering and anomaly detections techniques have been investigated [10, 11] and brought valuable results [12]. They allow the identification of flights that differ from the other ones from a statistical point of view. The review of such flights by experts could highlight new safety issues. Nevertheless these techniques have not been widely included in commercial tools. Advances in data-mining techniques are also used to search for precursors of adverse events [13]. The methodology supposes to have identified a set of nominal flights (without the adverse event) and a set of adverse flights (with the adverse event) for the learning process.

These new algorithms offer valuable insights in the operational reality but require a subject matter expert to evaluate the impact on safety and links with standard operating procedures.

\subsection{Crew activity identification}

A third way can be investigated to manage safety. Instead of describing what is outside safety margins (as prescribed exceedances) or considering "average" flight as references and search for atypicalities (roughly as data-mining tools), the idea is firstly to model the expected parameters evolutions, in terms of crew activity and aircraft attitudes and in relation with airlines procedures. Then the model can be used to better understand how crews apply these procedures, to find atypical achievement of specific procedures, or to set up the operational context of some exceedances (for example to make differences between unstabilized approaches according to the landing procedure used - Visual or ILS ${ }^{6}$ or Radar approach...). Also this third way is not opposed to the two other ones but has to be combined for a better understanding of operational hazards.

\footnotetext{
${ }^{6}$ Instrument Landing System
}

Section 3 is dedicated to the modeling of airlines' procedures and section 4 presents an application on GoAround ${ }^{7}$ procedures using real aviation data.

\section{MODELING FLIGHT OPERATING PROCEDURES}

\subsection{Airline and airport procedures}

Operating an aircraft for an airline is a complex task that requires many skills. While modern airplanes are highly automated and their systems can normally manage the flight, the flight crew has always a monitoring role which requires interacting with these highly automated systems. As commercial flights are highly standardized, normal, abnormal and emergency operations are described through procedures. Depending on the operational context, the crew is supposed to select and apply the appropriate procedure. Many of these procedures are defined by the aircraft manufacturer and adapted if necessary by the air carrier.

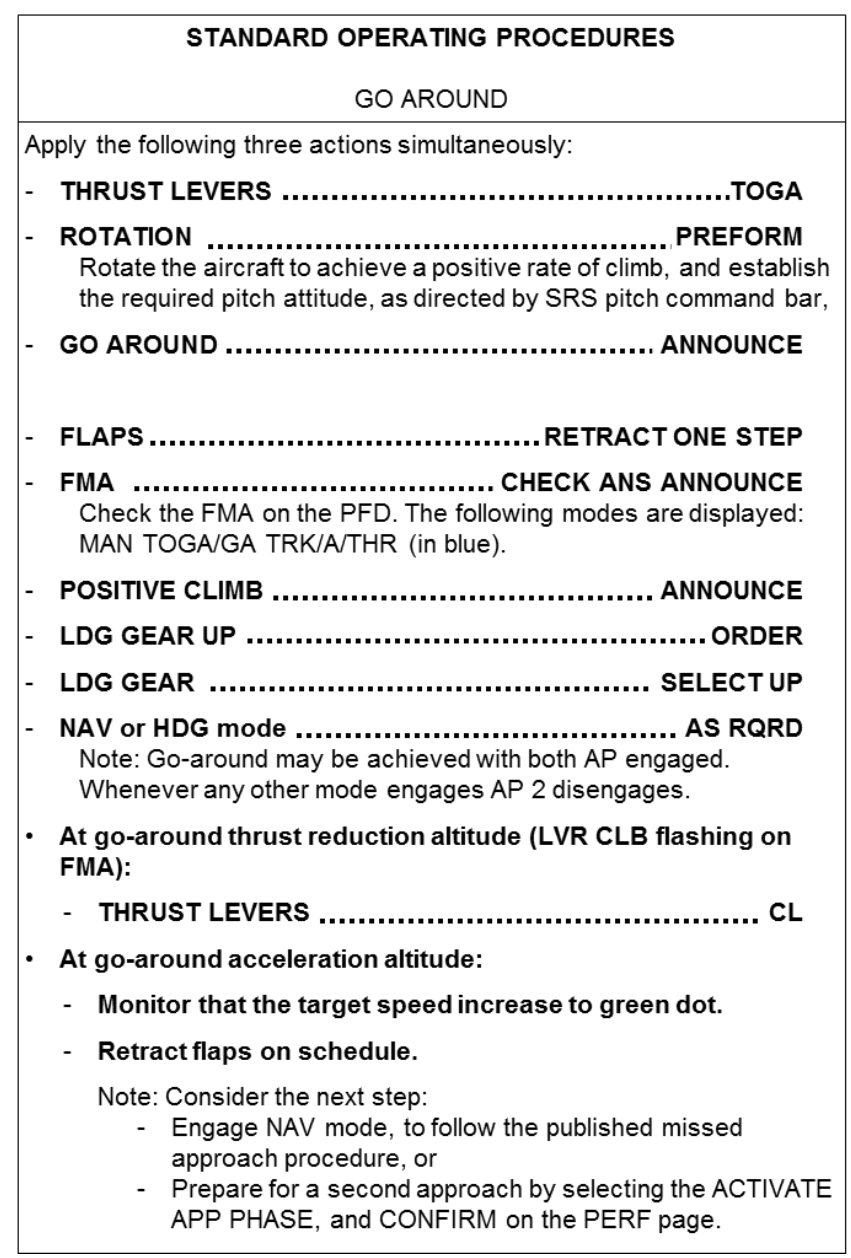

Figure 3: Generic go-around procedure for airbus aircraft. The figure represents neither the actual Airbus procedure nor a real airline procedure but is close from both of them. .

\footnotetext{
7 A go-around is defined as the sequence of manoeuvers executed by an aircraft to abort a landing attempt and recover a secure flight level to avoid losing safety.
} 
Figure 3 gives an example of such a procedure in which we can find physical actions (e.g. push the Thrust Levers to the Take-Off and Go-Around (TOGA) position), crew coordination (announce the Go-Around manoeuver) and monitoring actions (Check the Flight Mode Annunciator (FMA) which gives the state of the autopilot). Such a procedure specifies the sequence of actions that should be made to conduct a go-around.

But implementing these actions will also require further information to take into account contextual elements: air traffic controller clearances, weather, airport configuration, aircraft constraints (i.e. "retract flaps on schedule" refers to airspeed limitations for each flaps position) and state of its systems. While the airline SOP holds true for all go-around manoeuvers, specific elements related to the airport and the runway used are defined in dedicated airport procedures. Figure 4 illustrates what can be found in the airport arrival procedure, including trajectory constraints (climb, turn left...).

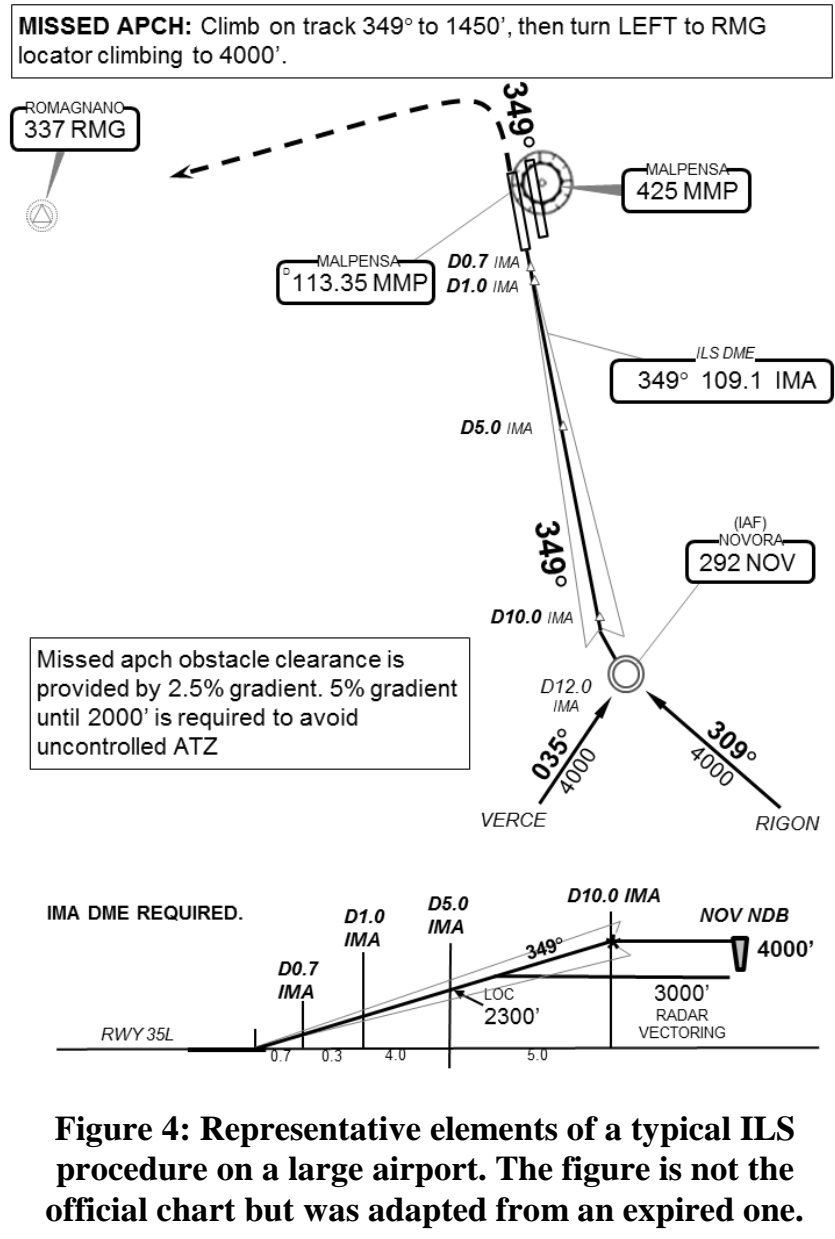

The number of available procedures is large as for example some airline can have 4 different go-around procedures to take into account the flight level at which the maneuver is started. Moreover each procedure can be adapted by the crew if required while SOPs cannot take into account all the specificities of the operational situation. Also, the idea is not to precisely model all the available procedures but to extract from them typical activity patterns which can be useful to set up a flight context and better identify and understand possible safety events occurring during flights.

\subsection{Crew activity profiles}

The leading idea is to use SOPs and other procedure elements to give a more precise operational context to flight sections. As an example, instead of knowing only that the aircraft is in final approach (as in current FDM tools), we could know which type of final approach is used (visual approach, ILS approach...) and in which part of the associated procedure the aircraft is (capturing or tracking the ILS slopes, doing the flare...). Having a more precise operational context, even if it will never be an exhaustive one, will help to better define new exceedances, to evaluate their safety impact but also to assess how the adherence to SOPs. Of course some deviations from SOPs can be justified by specific contextual factors (ATC clearances, weather...) but identifying them in a more systematic way will help safety officers to conduct analyses and decide which safety actions have to be implemented: modify, adapt or complete some procedures, improve the training process, add new exceedances to follow specific situations...).

Activity model - The use of elements described in SOPs or in training manuals to identify coherent flight portions requires first to represent them in an adequate model. Also, our work relies on a language dedicated to model the crew activity. The approach adopted to represent these activities is inspired by the script definition given by Schank and Abelson [14]: "A script, as we use it, is a structure that describes an appropriate sequence of events in a particular context... For our purpose, a script is a predetermined, stereotyped sequence of actions that define a well-known situation". The resulting language, called LDA (a French acronym standing for Activity Description Language), has been shortly presented in [15] and more completely specified in [16].

Roughly, the LDA is based on a hierarchical representation of the activity. The higher level is called a "Phase" and is used to model a significant part of the flight, as for example the descent or the final approach or the whole go-around phase. It can normally be associated with a crew high level objective. Often, there are several possibilities allowing completing this task. In other words, there are several stereotyped sequences of events usable to fulfil the goal. Each of them will be captured by a crew activity "Profile". So a profile is a specific sequence of actions that can be used to reach the objectives of the phase.

Each profile contains a sequence of events (or actions) representing this particular "way of doing" that can be used. These events that compose the profile are called 'Schemas'. For example, one profile representing the Final Approach phase may contain the three following schemas: "Align the runway", "Final descent", and "Flare" that represent the three main goals for the crew during this flight phase. Then, each "Schema" can contain more elementary actions, called 
"Modules" that represent how the "Schema" is implemented by the crew. Modules are the lowest level of actions taken into account by the LDA language and should represent elementary activities which make sense for the task captured by the Schema. For example, inside an ILS final approach, the schema "Align the runway" could contain at least three modules: "Follow a converging segment", "Capture the Localizer" and, "Capture the Glide Slope". Building a representation of the target activity from the SOPs and other materials is a complex task which requires a good understanding of the crew activity.

Temporal constraints can also be associated with modules and schemas, allowing the specification of sequences of events. Figure 5 shows a hypothetical flight phase $\mathrm{X}$ for which two ways of doing have been described. Profile 1 displays the first one for which three sequential schemas are expected. While the first schema (1.1) contains two Modules that can be made in parallel, the second schema is directly considered as an elementary action and so has no decomposition in modules. In the second profile, which describes the other way of doing, the second schema (2.2) can start before the end of the first one.

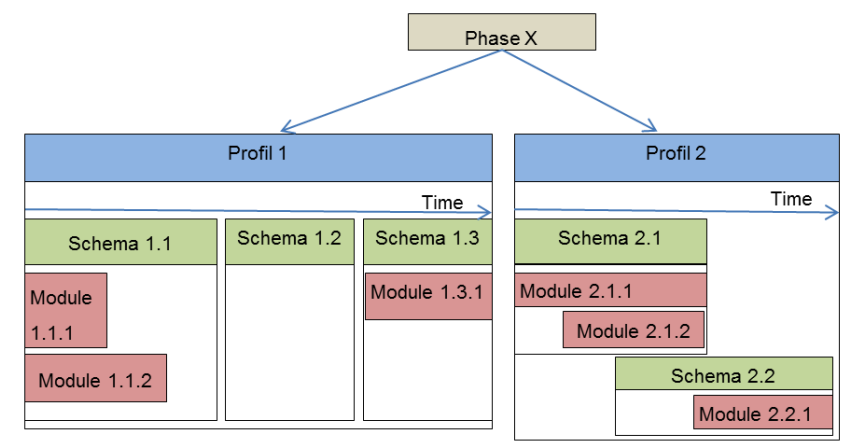

Figure 5: Example of crew activity representation with the LDA

From recorded data to activity - The next step is to be able to identify which profile has been used in a given flight through the analysis of the time evolution of the flight parameters. As the flight profile is mainly characterized by its composing schemas and modules, the first stage is to be able to identify these sub-elements. The approach adopted relies firstly on rules defining specific points (key points) in the flights (such as the touch down) from the evolution of the flight parameters.

Roughly, each key point is defined by a temporal window which specifies in which part of the flight to search for this key point, an identification rule (with dedicated thresholds) based on the evolution of flight parameters and the search direction. The description of the key point is made thanks to a xml file. As an example, a key point corresponding to the touch down can be defined with:

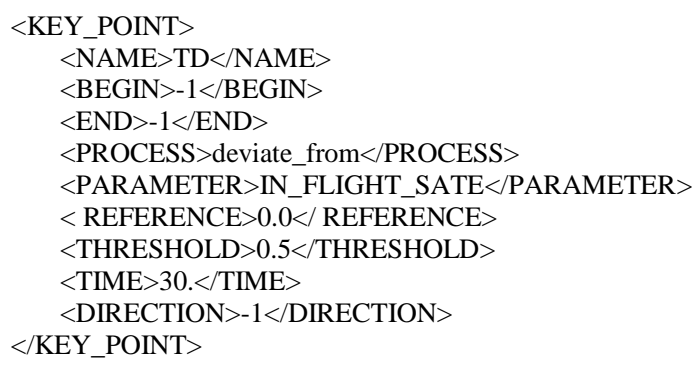

This description indicates that the key point named TD has to be searched on the whole flight (no constraint $(-1)$ for the beginning and no constraint for the end; names of already found key points can be used). The rule "deviate_from" has to be applied on the "IN_FLIGHT_STATE" parameter (which is one of the flight recorded parameters whose value is 0 when the aircraft is on the ground and 1 when the aircraft is in the air) with a reference value of 0 and a threshold of 0.5 . The search has to be done backward (because direction is set to -1), from the end of the search window to the beginning. The key point is validated only if the value of the parameter is over 0.5 for more than $30 \mathrm{~s}$ as the "TIME" is set to 30 (see figure 6).

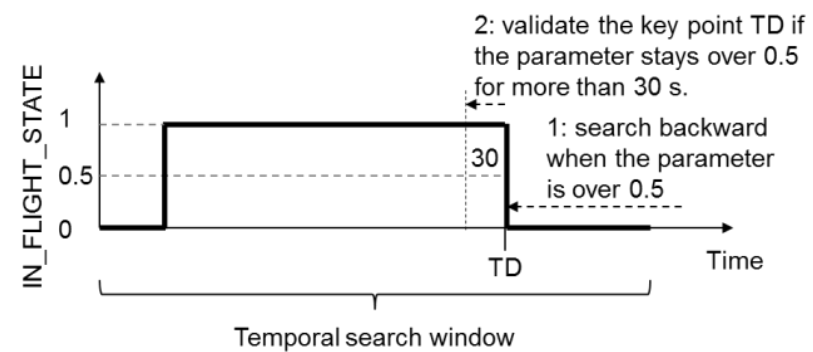

Figure 6: Determination of the TD key point with the "deviate_from" rule

The backward search is possible because the flight safety analysis is made when the flight is over. It has to be noticed that the take-off could also be easily identified using the same rule with a forward search. In the tool used for this study, 8 generic rules (as "deviate_from", "close-to"...) were defined.

Once all the defined key-points have been searched (some of them have perhaps not been found), these key points are used to identify modules, schemas, profiles and phases in the flight. This activity identification process uses an xml description of the hierarchical structure of modules and schemas that compose the profile. Each element is defined by two key-points identifying the beginning and the end of the element and a status flag that indicates if the element is required, optional or forbidden. Here is an example of a profile description where the profile starts at the FA keypoint and finishes at the TD key-point. The profile is composed of the schema "ILS Capture" that contains the two modules "Localizer Capture" and "Glide Capture". All these elements are required, so all their defining key-points have to exist in order to identify the activity. Figure 7 shows a sequence of key-points that allow the identification of the profile. 

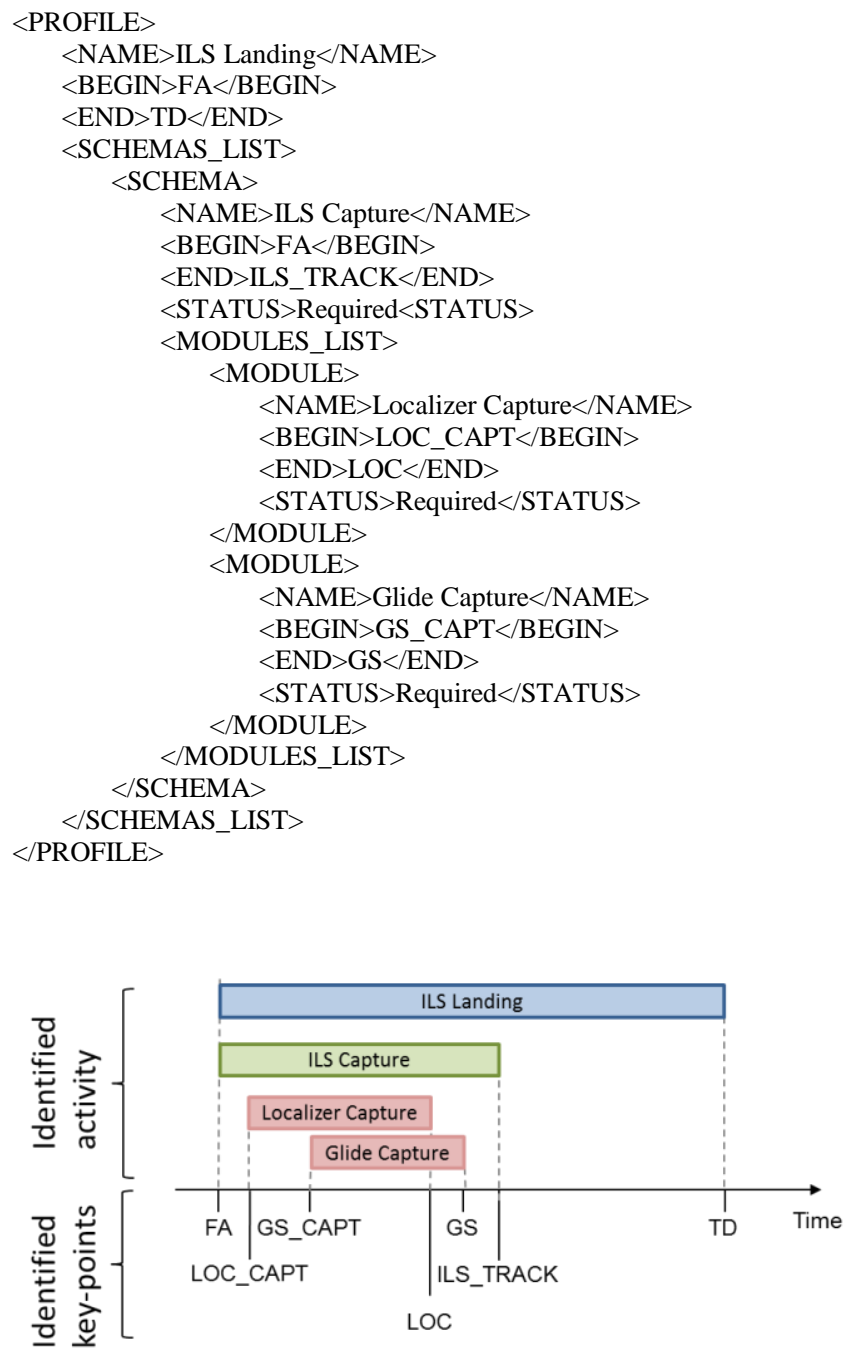

Figure 7: Example of crew activity identification (Pink: modules, Green: Schema, Blue: Profile)

\section{APPLICATION TO GO-AROUND PROCEDURES}

Let us now apply this methodology to model and identify go-around situations using aviation data provided by an air carrier. All go-around flights were selected from the database of airbus A319 flights landed in five major European airports over two years. It resulted in a set of more than 700 go-around flights. Each data record contains the time evolution of over 300 parameters (latitude, longitude, altitude, speed, autopilot modes, auto-thrust mode, flap and slat positions, landing gear position...) recorded at a frequency of $1 \mathrm{~Hz}$.

\subsection{Go-around profiles}

The standard operating procedures (cf. Figure 3 ) used by the airline were used to build the go-around profile. It was selected to emphasize in this study the automation level used by the crew to achieve the go-around maneuver as it is a relevant cue to safety management. So a set of four profiles was defined, with one dedicated to capture goaround maneuvers that do not start with the setting of the thrust levers to the take-off and go-around (TOGA) position:

L1: TOGA full automation. The go-around manoeuver relies on the autopilot and auto-thrust automation. The crew never directly flies the aircraft but monitors the automation and manages the aircraft configuration.

L2: Automated TOGA. The go-around maneuver is initiated with automated autopilot or auto thrust modes but the crew takes over control of the plane during the procedure.

L3: Manual TOGA. The crew manually controls the aircraft (with the side sticks) when the go-around maneuver is initiated. Automated modes can be engaged afterwards.

L4: Without TOGA detent. The landing attempt is aborted but without pushing throttles to TOGA detent.

Hierarchical structures of modules and schemas that compose each profile are very similar and in this paper we present only the one of profile L1 (see figure 8).

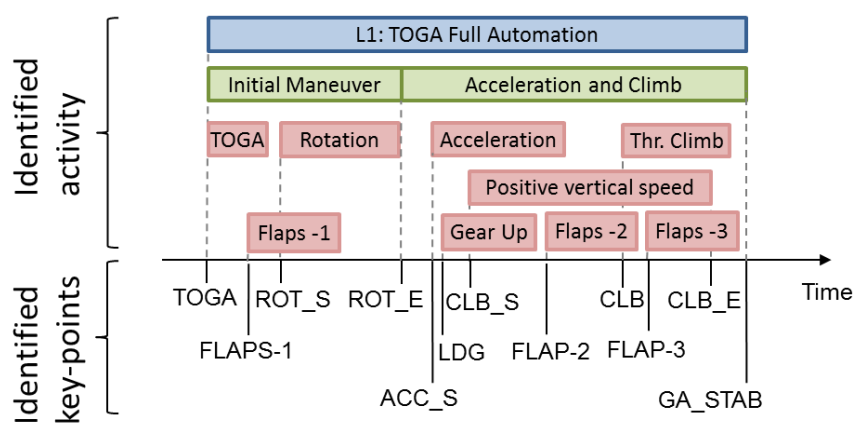

Figure 8: Hierarchical structure of profile L1 (Pink: modules, Green: Schema, Blue: Profile)

\subsection{Identified profiles in the flights}

Based on the definition of these four profiles, the XML files defining all the relevant key points (from the evolution of the recorded parameters) and all the activity elements were built. Then all the flights were processed by a dedicated tool (called ASVM, see figure 9) which automatically identifies for each flight the go-around procedure used and all the related schemas and modules. The identified activity is reported in an xml file which can be used for statistics, for adding information on a flight parameter display (as on Figure 10) or to extract focused parts of the flight for further analysis. 


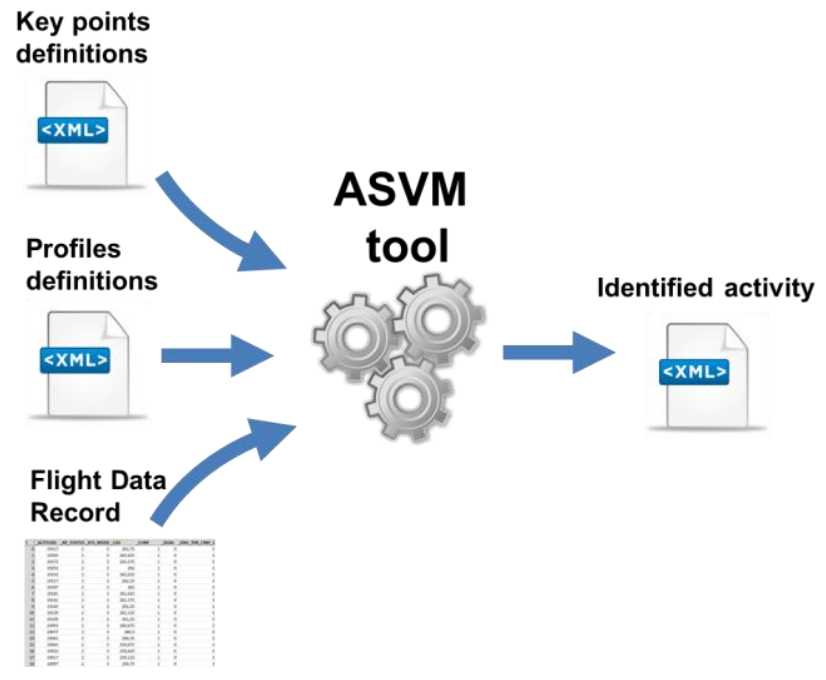

Figure 9: ASVM framework showing the inputs and output of the profile identification process.

Figure 10 displays the result for a specific flight where the go-around was initiated at $455 \mathrm{Ft}$ when the pilot pushed the throttle from the Climb position (parameter ENG_THR_CMD_1=3) to the TOGA detent. Nevertheless the pilot moved back the throttle to the Climb position 7 seconds later, just after the first flaps retraction. The pilot kept engaged the autopilot and the auto-thrust during the entire maneuver and so the L1 profile was recognized (TOGA full automation). It can be noticed that the pilot retracted only two steps of flaps (_CONF parameter).

The profile used to manage the go-around maneuver and all its constituting elements were identified for all the flights. Then, an unbiased view of how crews rely on automation to manage go-around maneuvers can be extracted. Figure 11 shows that in many cases the crew uses a full automation strategy (profile L1). Nevertheless, profile L3 is also often used. In this case the crew took manually the control of the airplane before deciding to abort the landing. Profile 2 is nearly never used ( $2 \%$ of flights) indicating that crews decide before initiating the go-around if they want to manually fly the aircraft but rarely disconnect the automation after the beginning of the maneuver. Profile 4 represents $12 \%$ of the analyzed flights so in practice goarounds are quite regularly made without pushing the throttle lever to the dedicated TOGA position.

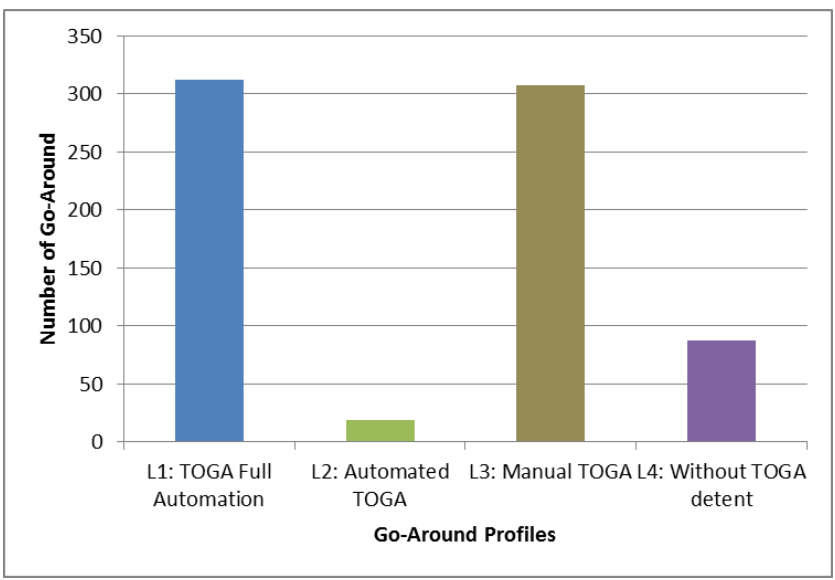

Figure 11: Breakdown of the flights with go-around by profiles

\section{NEW POSSIBILITIES FOR AVIATION SAFETY ANALYSIS}

In this section we discuss how such crew activity identification can contribute to enhance aviation safety analysis. The main idea is not to replace the actual exceedances based approach but rather to complement it by (1) the access to additional information on the adherence to SOPs, (2) the ability to use data exploration tools on more specific parts of flights and, (3) the possible definition of

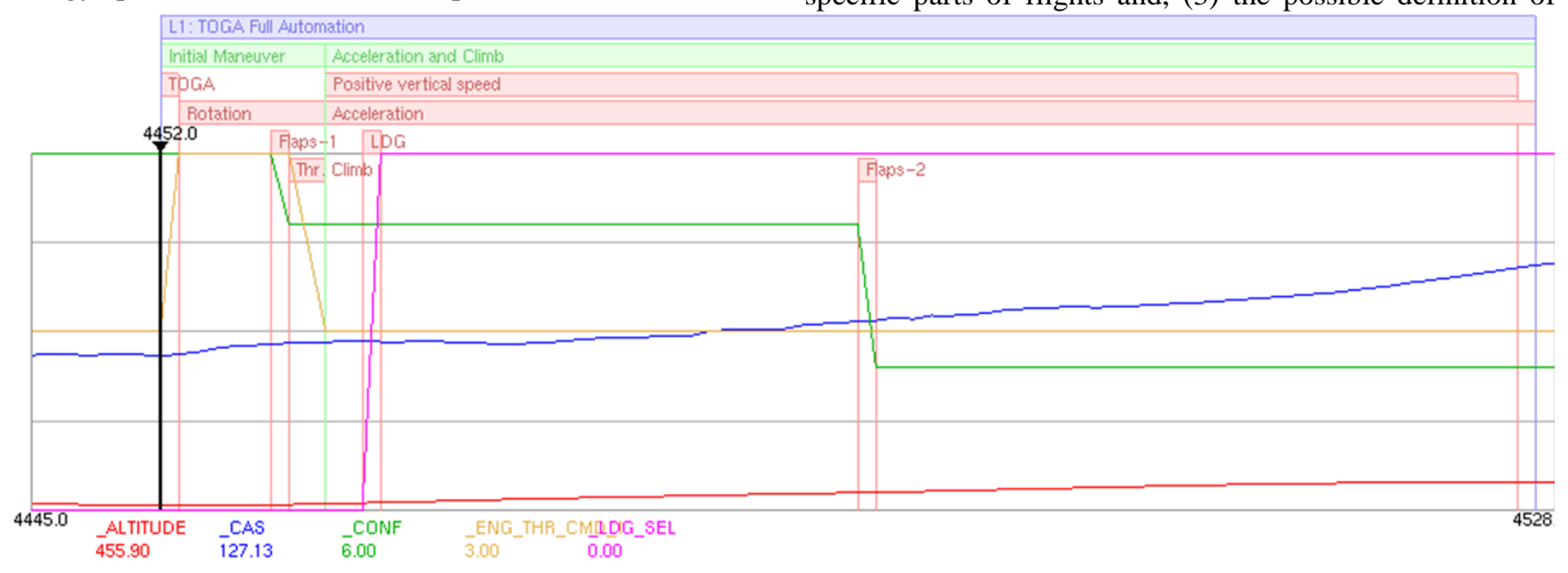

Figure 10: Activity identified and flight parameters evolution for one specific flight. (Red (_ALTITUDE): altitude above runway level in feet, Blue (_CAS): controlled air speed in knots, Green (_CONF): flaps and slats configuration, Khaki (_ENG_THR_CMD1): position of the engine 1 throttle, and Pink (_LDG_SEL): landing gear position (0 for down)) 
more specific exceedances. We will now review these three points.

\subsection{Adherence to SOPs}

The Airbus' Flight Operations Briefing Notes on Standard Operating Procedures [17] recalls that a strict adherence to suitable SOPs and normal checklists is an effective method to:

- Prevent or mitigate crew errors;

- Anticipate or manage operational threats; and thus,

- Enhance ground and flight operations safety.

Nowadays airline safety officers have only a limited view of how their flights adhere to SOPs. Of courses the defined exceedances used in their FOQA tools are closely related to SOPs but this exceedance based approach does allow neither the identification of which SOP was chosen by the crew, nor the real evaluation how the sequence of actions adhere to the selected SOP or to airline recommendations, nor the assessment of the suitability of the selected procedure.

As shown in the previous section, the analysis methodology described in this paper relies on the modeling and the identification of the procedure applied by the crew and also produces for each flight an understanding of which procedures were used. This is a first important step towards an analysis of the validity of the selected procedure. For example a flight safety officer could wonder if the profile L4, which is used in $12 \%$ of the studied flights, is always the most relevant one as the throttle lever is not pushed in the TOGA position. A first cue is given by the analysis of the altitude above the runway at the beginning of the goaround maneuver. As shown on figure 12, profile L4's goarounds are usually made at high altitude while profile L3's go-around are low level ones, often around the decision altitude defined by the procedure. Thus the selection of the procedure seems coherent with the airline standard while the specific TOGA throttle position is not used only in case of high altitude interrupted approaches.

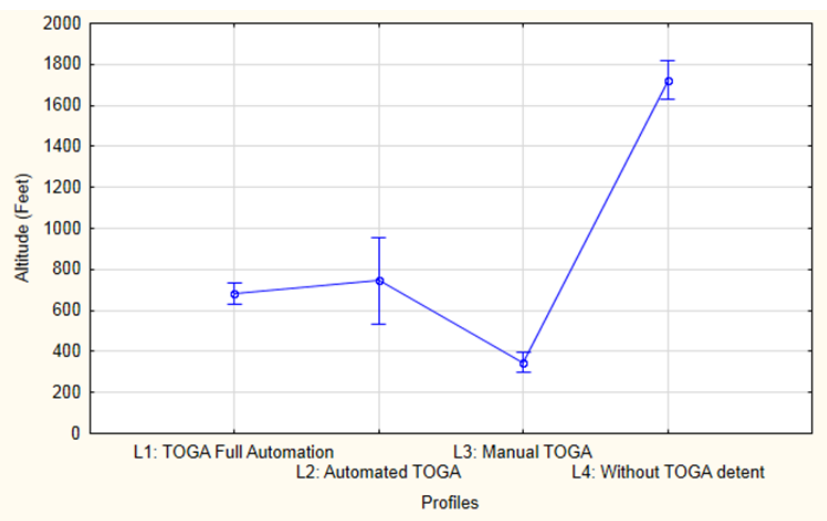

Figure 12: Mean altitude and confidence interval $(p=0.95)$ at the beginning of the go-around maneuver for each profile
Then, for each profile it is now possible to check if the sequence of action squares with the procedure. Let us for example focus on the aircraft flaps and landing gear management. The sequence of changes in the flaps and landing gear settings during the go-around maneuver can be extracted for each flight. Figure 13 summarizes all these sequences for the 439 flights that start the maneuver in a full extended flaps configuration with the landing gear down. The most used sequence is to first retract flaps to configuration 3 , then retract the landing gear (LDG Up), retract flaps to configuration $1 \mathrm{pF}$ and finally end with flaps completely retracted (Conf 0). 309 flights finished the goaround maneuver in that configuration while 120 other ended the maneuver with flaps set in configuration 1. It can also be noted that in 31 flights the crew retracted the landing gear before retracting flaps from one step.

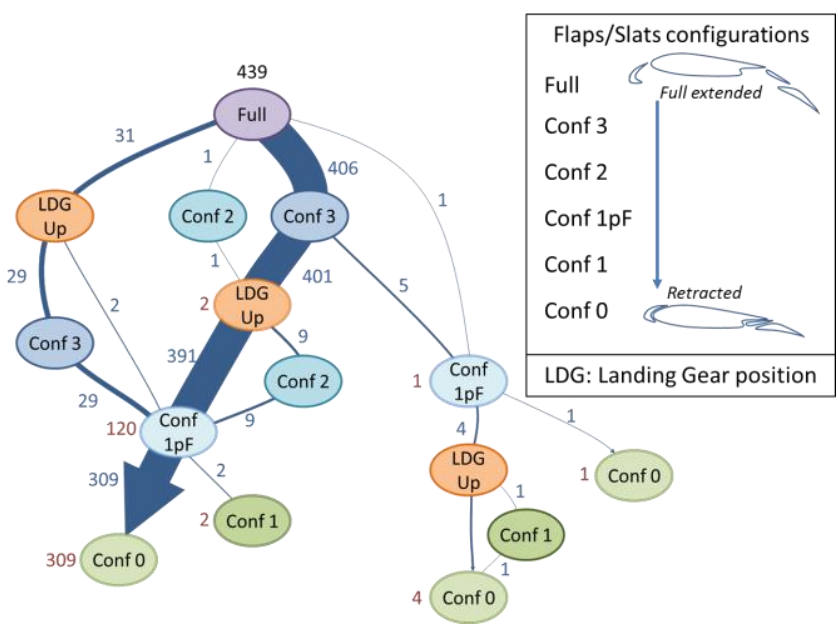

Figure 13: Sequences of flaps/Slats and landing gear changes. Blue numbers on lines indicate the number of flights that contain that specific sub-sequence. Red numbers are the numbers of flight ending the procedure in that configuration.

Also the identification of the procedure used with the extraction of the sequence of action made paves the way to a more circumstantial assessment of the adherence to SOPs. It can also highlight other "ways of doing" used in flight. All these elements should help to manage the training and perhaps the update of SOPs.

\subsection{Exploration tools}

The identification of the accomplishment of the procedure allows the extraction of the track of specific parameters during that period of time. Then several achievements of the procedure can be compared, either with graphical tools or with data-mining tools. Let us for example have a look at the evolution of the bank angle during the go-around procedure. Figure 14 displays the bank angle evolution from the initialization of the go-around to the end of the procedure (aircraft stabilized in altitude) for the whole set of studied flights. The color indicates the aircraft flaps and slats configuration while the figure has been generated with the FromDady tool [18]. 
Few flights with the Go-Around maneuver starting during a turn

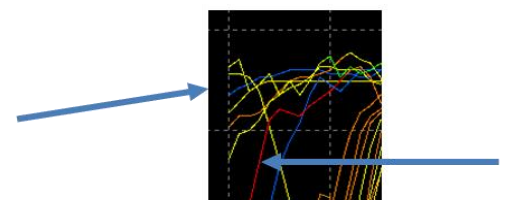

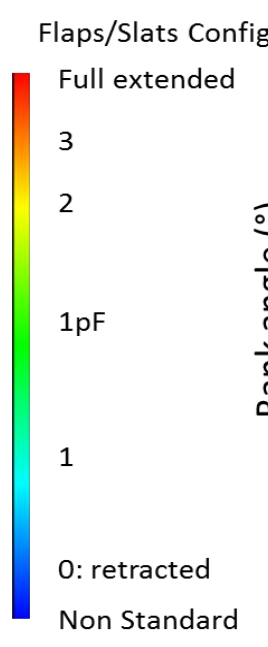

At least one flight

with a turn in the

full extended

configuration
Expected

bank angles

\section{Figure 14: Evolution of the bank angle during go-around procedures for 700 flights. Colors indicate flaps/slats configurations from full Retracted (blue) to full extended (red).}

On the upper-right figure a flight with a bank angle over $20^{\circ}$ and a flaps "full extended" configuration can be identified. This is a questionable maneuver as explained in the Gulf Air Flight GF-072 accident report [19]: "Flaps 'full' is a flapsetting intended only for the final phases of flight: approach and landing. It is generally selected when a landing can be accomplished. Due to the associated drag, flaps 'full' is not a setting for manoeuvring. A recommended setting for manoeuvring is flaps 'three', especially if the landing gear is 'down'. The effect of the high drag induced by the setting of flaps 'full' is to degrade the manoeuvrability of the aircraft. This typically results in exaggerated control inputs, or over-controlling, by the pilot." Thus such a visual examination of flight parameter tracks during a specific procedure can help to highlight flights that should be more specifically investigated.

Atypical achievement of the procedure can also be searched with more advanced data-mining tools [10, 11]. The extraction of the precise part of the flight, such as a procedure or an even more specific piloting activity, brings a more focused use of these tools. The MKAD tool [20] was applied on this set of data to rank the atypicality of the goaround procedure. Two kernels were used, one based on discrete data (autopilot status, lateral and longitudinal modes; auto throttle status and mode; flight director status; flaps configuration; landing gear position; engine throttle position) and the other one with continuous data (altitude; air speed; vertical speed; engines' N1 and N2 values; pitch; and bank angle). Figure 15 displays the evolution of some parameters of the more atypical achievement of the go- around procedure identified by this data mining tool (with these settings). The parameters that most contribute to the atypical score of this flight are: engine parameter N2, airspeed, throttle position and autopilot management parameters. It can be noted that the "initial maneuver" schema is particularly long (28s) with changes of the throttle position (between TOGA and FLX/MCT ${ }^{8}$ detent) and disconnection of both autopilots. Moreover, after the throttle lever was pushed back to the climb level and one autopilot reengaged the captain pushed again the throttle to the TOGA detent and disconnected the autopilot, as if he/she considered that the initial maneuver was not completely achieved. It can also be noted the late landing gear retraction (35 seconds after the initial TOGA action). The highlight of nonstandard flight management like this one has a high value for safety officers who want to investigate other safety risks than the one already captured by the prescribed exceedances of their FOQA tool.

Also this study contributes to demonstrate the value of using visualization and data mining tools on more specific parts of the flight.

\subsection{Specific exceedances}

The identification of the procedure used could also allow the definition of new exceedances, related to specific sequences of actions (flaps retraction before landing gear retraction in a go-around maneuver for example) or precise search

\footnotetext{
${ }^{8}$ MCT: Maximum Continuous Thrust.
} 


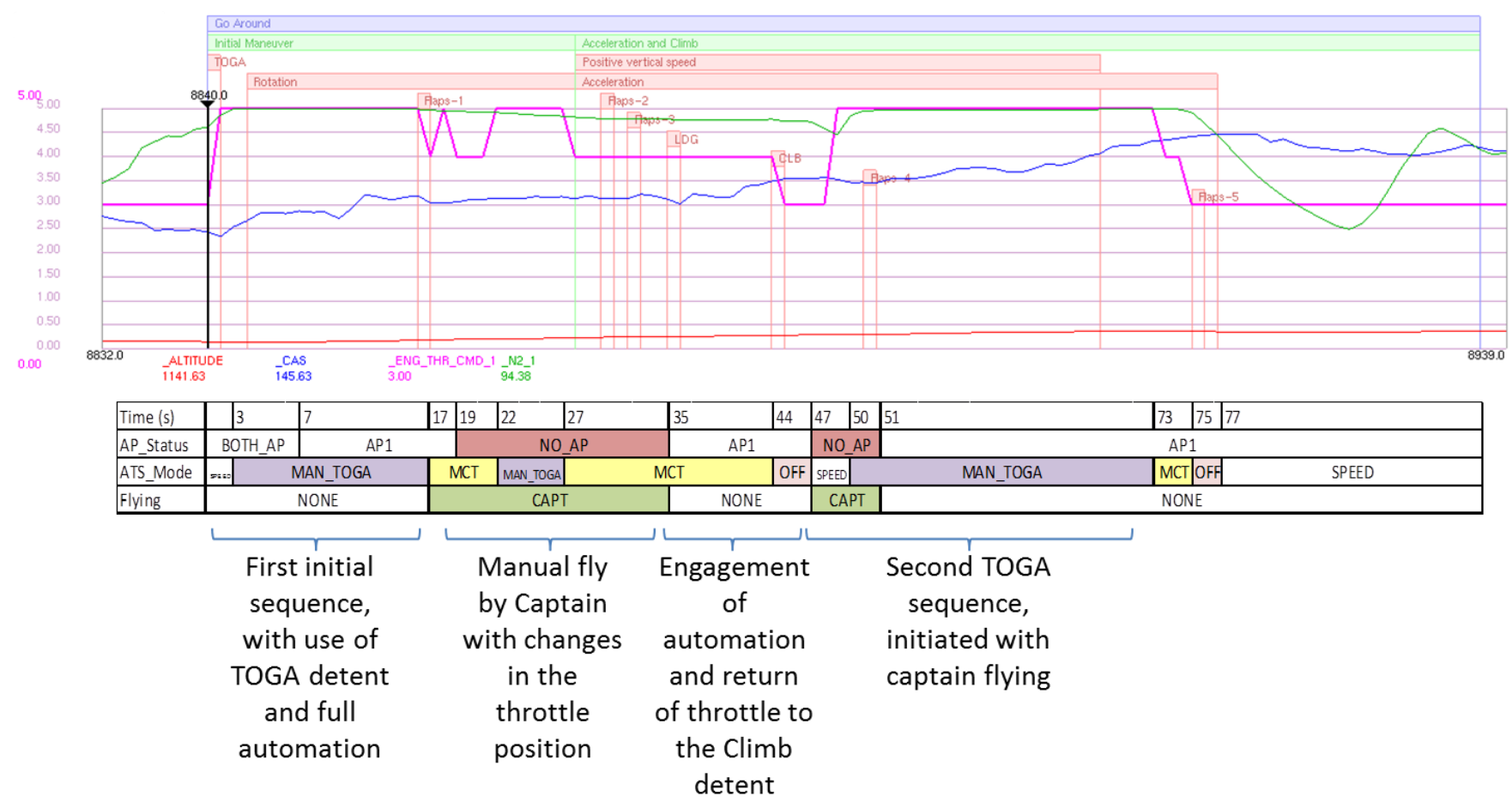

Figure 15: The most atypical go-around procedure achievement identified by the MKAD tool. The upper part shows the evolution of 4 parameters (Red: altitude in Feet, Blue: Airspeed in Knots, Pink: position of the engine 1 throttle, and Green: value of the N2 parameter for engine 1) while the lower part displays the autopilot status (AP_Status), the mode of the auto-thrust (ATS_Mode), and which member of the crew is manually flying the aircraft (Flying)

windows (turn during the initial go-around maneuver for example). The study of flights with unusual application of procedures is often inspiring for setting efficient new safety markers. Nevertheless this step has not been done yet with the airline.

\section{Conclusions}

This paper questions the process used to analyze inflight recorded data for safety purpose and suggest a new paradigm based on the modelling of airline procedures. An $\mathrm{xml}$ based language and an identification process are both described and applied to a go-around procedure. Based on the analysis of a set of 700 commercial flights with an interrupted landing, the study demonstrates the feasibility of the proposed methodology. Then the paper demonstrates that this new approach increases the understanding of how crew activity adheres to SOPs. This is an important step towards an adaption of the training and eventually the SOPs in order to better mitigate risks. Moreover this fine identification of crew behavior can be used to lead the use of other tools and we demonstrate that data mining tools can be efficiently applied on small portions of flight.

\section{REFERENCES}

[1] ICAO, 2011 State of Global Aviation Safety

[2] ICAO, Safety Report, 2016 Edition

[3] Wolf, H. 2012. The Emerging Role of Safety Management Systems in Aerospace. In IEEE Aerospace Conference, 3-10 March 2012, Big Sky, MT USA.

[4] ICAO, 2010. Annex 6: Operation of Aircraft, Seventh edition, Order Number: AN 6-3, ISBN 978-92-9231-5375.

[5] GAIN 2004. Role of Analytical Tools in Airline Flight Safety Management Systems, Global Aviation Information Network Report, Second Edition.

[6] Speyer, J.-J. 2002. The Flight Operations Monitoring System: A Bundled Approach for Synergistic Safety Management. In: Proceedings of the International Conference on Human-Computer Interaction in Aeronautics, AAAI, 23-25 October 2002, Menlo Park, CA USA.

[7] Jesse, C. 2007. FOQA/FDM in Times of Change - Case Studies. In: Proceedings of the 9th European Aviation Safety Seminar. 12-14 March 2007, Amsterdam, Netherlands. 
[8] Boeing, 2016. Statistical Summary of Commercial Jet Airplane Accidents. Worldwide Operations, 1959 - 2015.

[9] Pedro. Soares, 2014. Flight Data Monitoring and its Application on Algorithms for Precursor Detection. PhD These.

[10] B. G. Amidan and T. A. Ferryman. 2005. Atypical event and typical pattern detection within complex systems, IEEE Aerospace Conference Proceedings, pages 36203631 .

[11] Li, L., Das, S., Hansman, R., Palacios, R., Srivastava, A. 2015. Analysis of Flight Data Using Clustering Techniques for Detecting Abnormal Operations, In: Journal of Aerospace Information Systems, Vol.12, pp 587-598.

[12] N. Maille. 2013. On the use of data-mining algorithms to improve FOQA tools for Airlines. IEEE Aerospace Conference Proceedings.

[13] V. Janakiraman, B. Matthews and, N. Oza, 2016. Discovery of Precursors to Adverse Events using Time Series Data. SIAM International Conference on Data Mining Proceedings, pp 639-647, May 5-7 2016, Miami, Florida, USA.

[14] Schank, R., Abelson, R. 1977. Scripts, Plans, Goals, and Understanding: An Inquiry into Human Knowledge Structures, Hillsdale, NJ: Lawrence Erlbaum Associates.

[15] Bayssié, L., Chaudron, L., Le Blaye, P., Maille, N., Sadok, S. 2003. Human Activity Modeling for Flight Safety, In: Proceedings of the Intl. Conf. on Digital Human Modeling for Design and Engineering, 16 - 19 June 2003, Montreal, Canada

[16] Bonnet, P. 2008. Un langage de description de l'activité de pilotage, pour l'analyse systématique des vols et l'étude de la variabilité de l'activité. ONERA Report MF 1/13534 DCSD. In French.
[17] Airbus, 2006. Flight Operations Briefing Notes Standard Operating Procedure. FOBN Reference: FLT_OPS - SOP - SEQ 01 - REV 04 - SEP. 2006.

[18] Hurter, C., Tissoires, B., Conversy, S. 2009. FromDaDy: Spreading Aircraft Trajectories Across Views to Support Iterative Queries, In: IEEE Transactions on Visualization and Computer Graphics 15(6):1017-24, November 2009.

[19] BEA, 2000. Accident Investigation Report, Gulf Air Flight GF-072 on 23/08/2000.

[20] Das, S., Matthews, B., Srivastava, A., 2010. Multiple Kernel Learning for Heterogeneous Anomaly Detection: Algorithm and Aviation Safety Case Study. In: Proceedings of the $16^{\text {th }}$ ACM SIGKDD international conference on knowledge discovery and data mining, ACM, July 25-28, 2010, Washington, DC, USA.

\section{BIOGRAPHY}

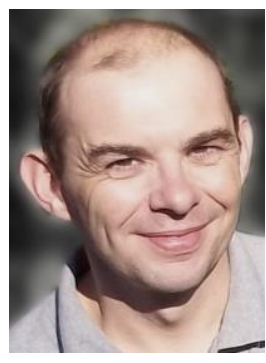

Nicolas Maille graduated from a French engineering school (Supaero) in 1993 and received a PhD thesis from Supaero in 1999. Since 2000, he has conducted researches at ONERA - The French Aerospace Lab, in the field of artificial intelligence and human factors. His activity includes the development of experience feedback methodologies and tools for aircraft operators, both civil and military. 